\title{
Robust mainlobe interference suppression for coherent interference environment
}

\author{
Yasen Wang ${ }^{*}$, Qinglong Bao and Zengping Chen
}

\begin{abstract}
A mainlobe interference suppression method is proposed in this paper, which can still work when the signal of interest (SOI) is present in the training data. In this method, the iterative adaptive approach (IAA) is applied to spatial spectrum estimation at first. Then, IAA spatial spectrum is used to reconstruct the interference-plus-noise covariance matrix (INCM). Next, the eigenvector associated with mainlobe interference in INCM is determined, and the eigen-projection matrix can be calculated to suppress the mainlobe interference. Meanwhile, the sidelobe-interference-plus-noise covariance matrix (SINCM) can be reconstructed. Finally, the adaptive weight vector is obtained. One main advantage is that the proposed method can deal with coherent mainlobe interference and sidelobe interferences simultaneously. Simulation results demonstrate the effectiveness and robustness of the proposed method.
\end{abstract}

Keywords: Mainlobe interference suppression, Iterative adaptive approach, Covariance matrix reconstruction, Eigen-projection

\section{Introduction}

Adaptive beamformers have wide applications in radar, navigation, sonar, radio astronomy, microphone array speech processing, and so on. Conventionally, adaptive beamformers are applied to suppress interferences that exist in sidelobe area $[1,2]$. This kind of interference is called sidelobe interference. However, when the interference from mainlobe area exists, it will induce peak offset and distortion of the mainlobe and heightening of the sidelobe level. This kind of interference is mainlobe interference. Nowadays, as the electromagnetic environment becomes more complex, the mainlobe interference appears more frequently. Alternative solutions such as the blocking matrix preprocessing (BMP) method [3] and the eigen-projection matrix preprocessing (EMP) method [4] for suppressing the mainlobe interference are proposed. However, the former could work well provided that the direction of mainlobe interference and giving rise to the reduction of the effective degrees of freedom, and the latter relies on the absence of the signal of interest (SOI) in the training data. Furthermore, the problem of mainlobe peak offset cannot be overcome

\footnotetext{
* Correspondence: wangyasen05@163.com

College of Electronic Science and Engineering, National University of Defense Technology, Changsha 410073, China
}

by both methods. Later, a modified EMP method [5] is proposed for solving the mainlobe peak offset problem. Another new method based on EMP method and similarity constraints [6] can suppress multiple mainlobe interferences. Both of them make the assumption that there is no SOI appearing in the training data, which is not always available in many applications such as passive location, mobile communications, and radio astronomy [7]. For example, in the case of passive radar, the transmit signal may already exist when the receiver starts working. Besides, coherent interference is another problem in adaptive beamforming. In applications like passive radar [8] and meter-wave radar [9], it is likely that target echoes are masked by multipath echoes, which would lead to coherent interference. When this coherent interference appears in mainlobe area, the methods mentioned above are no longer effective.

Recently, some new beamforming techniques have been proposed [10-12]. Among them, an interferenceplus-noise covariance matrix (INCM) reconstruction processing is put forward in [8] to eliminate the SOI component when calculating the adaptive weight vector. However, the performance of this procedure degrades when coherent interference exists. In [13] and [14], an iterative adaptive approach (IAA) is proposed, which has 
high accuracy of direction of arrival (DOA) and power estimation even when coherent sources and finitesampling effects exist. In this paper, the IAA spatial spectrum is used to reconstruct the INCM. Then, inspired by the method proposed in [5], mainlobe and sidelobe interferences can be suppressed using the reconstructed INCM. Compared to the existing methods, the proposal in this paper can handle the coherent mainlobe interference even when the SOI is present in the training data.

\section{Signal model}

Consider an array composed of $M$ omnidirectional antenna elements, the output of a narrowband adaptive beamformer at the $k$ th snapshot $y(k)$ is given by $y(k)=$ $\mathbf{w}^{H} \mathbf{x}(k)$, where $\mathbf{w} \in C^{M \times 1}$ is the complex beamforming weight vector, $\mathbf{x}(k)$ stands for the signal received by the array antenna, and $(.)^{H}$ denotes the Hermitian transpose. Assume there are $Q$ sidelobe interferences and one mainlobe interference, where $Q+1<M$; then, $\mathbf{x}(k)$ is given by

$$
\begin{aligned}
\mathbf{x}(k) & =\mathbf{x}_{s 0}(k)+\mathbf{x}_{\mathrm{int}}(k)+\mathbf{n}(k) \\
& =\sum_{q=0}^{Q+1} \mathbf{a}\left(\theta_{q}\right) s_{q}(k)+\mathbf{n}(k)
\end{aligned}
$$

where $s_{q}(k)$ and $\mathbf{a}\left(\theta_{q}\right)$ are the $q$ th signal waveform and the corresponding steering vector (SV), respectively. $\theta_{q}$ stands for the qth signal DOA, and $\mathbf{n}(k)$ denotes the additive white Gaussian noise. $\mathbf{x}_{s 0}(k)=\mathbf{a}\left(\theta_{0}\right) s_{0}(k)$ and $\mathbf{x}_{\text {int }}$ $(k)=\sum_{q=1}^{Q+1} \mathbf{a}\left(\theta_{q}\right) s_{q}(k)$ denote the SOI and interference vectors, respectively. $s_{Q+1}(k)$ denotes the mainlobe interference waveform. The INCM can be written as

$$
\mathbf{R}_{i+n}=\mathrm{E}\left\{\left(\mathbf{x}_{\text {int }}(k)+\mathbf{n}(k)\right)\left(\mathbf{x}_{\text {int }}(k)+\mathbf{n}(k)\right)^{H}\right\} \in C^{M \times M}
$$

The solution to the minimum variance distortionless response (MVDR) beamformer is given by

$$
\mathbf{w}_{\mathrm{opt}}=\frac{\mathbf{R}_{i+n}^{-1} \mathbf{a}\left(\theta_{0}\right)}{\mathbf{a}\left(\theta_{0}\right)^{H} \mathbf{R}_{i+n}^{-1} \mathbf{a}\left(\theta_{0}\right)}
$$

Since $\mathbf{R}_{i+n}$ is unavailable when the SOI is present in the training data, it is usually replaced by the sample covariance matrix $\hat{\mathbf{R}}=\frac{1}{K} \sum_{k=1}^{K} \mathbf{x}(k) \mathbf{x}^{H}(k)$, where $K$ is the number of snapshots. Then, the adaptive beamformer $\mathbf{w}_{\text {SMI }}=\frac{\hat{\mathbf{R}}^{-1} \mathbf{a}\left(\theta_{0}\right)}{\mathbf{a}\left(\theta_{0}\right)^{H} \hat{\mathbf{R}}^{-1} \mathbf{a}\left(\theta_{0}\right)}$ is the sample matrix inversion (SMI) beamformer. The corresponding Capon spatial spectrum distribution can be calculated as

$$
\hat{P}_{\text {Capon }}(\theta)=\frac{1}{\mathbf{d}^{H}(\theta) \hat{\mathbf{R}}^{-1} \mathbf{d}(\theta)}
$$

where $\mathbf{d}(\theta)$ is the SV associated with the array structure and a hypothetical direction $\theta$. Whenever the training data contains the SOI, SMI beamformer is in essence a minimum power distortionless response (MPDR) beamformer. However, the MPDR beamformer is more sensitive than MVDR beamformer when SV error exists [15].

\section{Proposed method}

In this paper, a mainlobe coherent interference suppression algorithm is proposed based on IAA spatial spectrum, INCM reconstruction, eigen-projection processing, and sidelobe-interference-plus-noise covariance matrix (SINCM) reconstruction.

\subsection{IAA algorithm}

IAA is a nonparametric adaptive algorithm. This algorithm assumes the presence of a large number of potential targets uniformly located on a fine grid with $P \gg M$ points in it. Let $\mathbf{P}$ be a $P \times P$ diagonal matrix whose diagonal contains the power at each angular grid point. Besides, define $\mathbf{A}(\theta) \triangleq\left[\begin{array}{llll}\mathbf{a}_{1} & \mathbf{a}_{2} & \cdots & \mathbf{a}_{P}\end{array}\right]$ with $\mathbf{a}_{p}$ denoting the SV of the $p$ th source and $\mathbf{s}(k) \triangleq\left[\begin{array}{llll}s_{1}(k) & s_{2}(k) & \cdots & s_{P}(k)\end{array}\right]^{T}$ is the source waveform vector. Define the interference and noise covariance matrix $\mathbf{G}_{p}$ to be $\mathbf{G}_{p}=\mathbf{R}-\left|s_{p}\right|^{2} \mathbf{a}_{p} \mathbf{a}_{p}^{H}$ where $\mathbf{R}=\mathbf{A} \mathbf{P A}{ }^{H}$; then, the cost function is given by

$$
\sum_{k=1}^{K}\left\|\mathbf{y}(k)-s_{p}(k) \mathbf{a}_{p}\right\|_{\mathbf{G}_{p^{-1}}}^{2}
$$

where $\|\mathbf{x}\|_{\mathbf{G}_{p^{-1}}}^{2} \triangleq \mathbf{x}^{H} \mathbf{G}_{p^{-1}} \mathbf{x}$. The minimization of (5) leads to the IAA algorithm. The initialization of IAA is done by the data-independent delay-and-sum (DAS) beamformer. The steps of IAA can be summarized as follows.

Initialization:

$$
\begin{gathered}
\text { Set } \hat{s}_{p}(k)=\mathbf{a}_{p}^{H} \mathbf{y}(k) / M, p=1, \cdots, P, k=1, \cdots, K \\
\hat{P}_{p}=1 / K \sum_{k=1}^{K}\left|\hat{s}_{p}(k)\right|^{2}, p=1, \cdots, P
\end{gathered}
$$

Iteration:

$$
\begin{aligned}
& \begin{aligned}
\overline{\mathbf{R}} & =\mathbf{A}(\theta) \hat{\mathbf{P}} \mathbf{A}^{H}(\theta) \\
\text { for } p & =1, \cdots, P \\
\mathbf{w}_{p} & =\frac{\overline{\mathbf{R}}^{-1} \mathbf{a}_{p}}{\mathbf{a}_{p}^{H} \overline{\mathbf{R}}^{-1} \mathbf{a}_{p}}, \quad \hat{P}_{p}=\mathbf{w}_{p}^{H} \hat{\mathbf{R}} \mathbf{w}_{p}
\end{aligned}
\end{aligned}
$$

end for

Termination:

$\hat{\mathbf{p}}^{i}$ represents the vector containing the power estimates at the $i$ th iteration in IAA algorithm. Thus, the iteration is 
terminated when the relative change $\left\|\hat{\mathbf{p}}^{(i)}-\hat{\mathbf{p}}^{(i-1)}\right\|_{F}$ is less than a specified tolerance, where $\|\cdot\|_{F}$ denotes the Frobenius norm.

An accurate spatial spectrum can be obtained from the results of IAA, and the SOI DOA $\hat{\theta}_{0}$ is also determined by searching for the peak of IAA spatial spectrum in the possible angle sector. Besides, the performance of IAA is not affected much by the presence of coherent sources [14]. For each IAA iteration, the complexity is $O\left(M^{2} P\right)$. Thus, a bigger $P$ results in a higher accuracy as well as a larger calculation.

\subsection{INCM reconstruction}

According to [7], INCM can be reconstructed as

$$
\hat{\mathbf{R}}_{i+n}=\int_{\bar{\Theta}} \hat{P}_{\text {Capon }}(\theta) \mathbf{d}(\theta) \mathbf{d}^{H}(\theta) d \theta
$$

where $\Theta$ is the angular sector in which only the desired signal is located and $\bar{\Theta}$ denotes the complement sector of $\Theta$. When mainlobe interference exists, it also locates in $\bar{\Theta}$. $\Theta$ is easily determined by the results of IAA spatial spectrum. Then, the INCM can be reconstructed as

$$
\hat{\mathbf{R}}_{i+n}=\sum_{\theta_{p} \in \bar{\Theta}} \hat{P}_{p} \mathbf{d}\left(\theta_{p}\right) \mathbf{d}^{H}\left(\theta_{p}\right)
$$

where $\hat{P}_{p}$ is obtained through IAA.

\subsection{Eigen-projection processing}

The reconstructed INCM is eigen-decomposed as

$$
\hat{\mathbf{R}}_{i+n}=\sum_{i=1}^{M} \lambda_{i} \mathbf{e}_{i} \mathbf{e}_{i}^{H}=\mathbf{E}_{s} \boldsymbol{\Lambda}_{s} \mathbf{E}_{s}^{H}+\mathbf{E}_{n} \boldsymbol{\Lambda}_{n} \mathbf{E}_{n}^{H}
$$

where $\left\{\lambda_{i}, i=1, \cdots, M\right\}$ is the eigenvalues of $\hat{\mathbf{R}}_{i+n}$ in the descending order and $\mathbf{e}_{i}$ is the $i$ th eigenvector. $\mathbf{E}_{s}=\left[\mathbf{e}_{1}, \mathbf{e}_{2}\right.$, $\left.\cdots, \mathbf{e}_{Q+1}\right]$ spans the interference subspace and $\mathbf{E}_{n}=\left[\mathbf{e}_{Q+2}\right.$, $\left.\cdots, \mathbf{e}_{M}\right]$ spans the noise subspace. $\boldsymbol{\Lambda}_{s}=\operatorname{diag}\left[\lambda_{1}, \cdots, \lambda_{Q+1}\right]$ and $\boldsymbol{\Lambda}_{n}=\operatorname{diag}\left[\lambda_{Q+2}, \cdots, \lambda_{M}\right]$.

The estimation of $Q$ is a public problem and is very critical. For an array with known array structure and SOI DOA, the mainlobe width is fixed. For example, for a uniform linear array (ULA), the mainlobe width $\mathrm{BW}_{0}$ can be expressed as

$$
\mathrm{BW}_{0}=2 \arcsin \left(\frac{\lambda}{M d}+\sin \theta_{0}\right)
$$

Therefore, the mainlobe angle sector $\Phi$ can be defined as

$$
\Phi=\left(\theta_{0}-{ }^{\mathrm{BW}_{0}} / 2, \theta_{0}+{ }^{\mathrm{BW}} / 2\right)
$$

$\bar{\Phi}$ denotes the complement sector of $\Phi$. By using $\bar{\Phi}$ and the results of IAA spatial spectrum, the number of sidelobe interferences $Q$ can be determined. Besides, when more than one mainlobe interference exists, the number of mainlobe interference can also be determined through the same method.

The correlation coefficient of two vectors $\mathbf{x}_{1}$ and $\mathbf{x}_{2}$ is defined as $\operatorname{cor}\left(\mathbf{x}_{1}, \mathbf{x}_{2}\right)=\frac{\mathbf{x}_{1}^{H} \mathbf{x}_{2}}{\left\|\mathbf{x}_{1}\right\| \cdot\left\|\cdot \mathbf{x}_{2}\right\|}$. Then, the correlation coefficients of SOI SV $\mathbf{a}\left(\hat{\theta}_{0}\right)$ and interference eigenvectors $\mathbf{e}_{i}(i=1, \cdots, Q+1)$ are calculated. These correlation coefficients can be maximized when the interference is a mainlobe interference. Therefore, the mainlobe interference eigenvector $\mathbf{e}_{m}$ can be determined as

$$
\left|\operatorname{cor}\left(\mathbf{e}_{m}, \mathbf{a}\left(\hat{\theta}_{0}\right)\right)\right|=\max _{\mathbf{e}_{i}}\left|\operatorname{cor}\left(\mathbf{e}_{i}, \mathbf{a}\left(\hat{\theta}_{0}\right)\right)\right|
$$

Then, the corresponding eigenvalue $\lambda_{m}$ can be obtained. When multiple mainlobe interferences like J mainlobe interferences exist, $\mathbf{e}_{m}$ can be determined by searching for the biggest $\mathrm{J}$ values of the correlation coefficients.

The eigen-projection matrix can be calculated as

$$
\hat{\mathbf{B}}=\mathbf{I}-\mathbf{e}_{m}\left(\mathbf{e}_{m}^{H} \mathbf{e}_{m}\right)^{-1} \mathbf{e}_{m}^{H}
$$

where $\mathbf{I}$ is the identity matrix. $\hat{\mathbf{B}}$ is adopted to suppress the mainlobe interference in echo data $\mathbf{X}$ as follows

$$
\mathbf{Y}=\hat{\mathbf{B}} \mathbf{X}
$$

\subsection{SINCM reconstruction}

$\lambda_{d}$ represents the average value of the noise eigenvalues. In order to remove the effect of background noise perturbation, $\lambda_{d}$ is used to replace the noise eigenvalues. $\lambda_{d}$ can be obtained by

$$
\lambda_{d}=\hat{\sigma}_{n}^{2}=\frac{\lambda_{Q+2}+\cdots+\lambda_{M}}{M-Q-1}
$$

$\lambda_{m}$ is the eigenvalue corresponding to $\mathbf{e}_{m}$. According to [4] and [3], a noticeable part of mainlobe interference will be eliminated if its eigenvalue $\lambda_{m}$ is replaced by $\lambda_{d}$. Thus, the SINCM can be reconstructed as

$$
\hat{\mathbf{R}}_{\mathrm{re}}=\mathbf{E} \hat{\mathbf{\Lambda}} \mathbf{E}
$$

where $\hat{\boldsymbol{\Lambda}}=\operatorname{diag}\left\{\lambda_{1}, \cdots, \lambda_{m-1}, \hat{\sigma}_{n}^{2}, \lambda_{m+1}, \cdots, \lambda_{Q+1}, \hat{\sigma}_{n}^{2}, \cdots, \hat{\sigma}_{n}^{2}\right\}$ is the reconstructed eigenvalue matrix and the eigenvector matrix $\mathbf{E}=\left[\begin{array}{ll}\mathbf{E}_{s} & \mathbf{E}_{n}\end{array}\right]$ remains unchanged.

Based on the reconstructed SINCM $\hat{\mathbf{R}}_{\text {re }}$, the weight vector of the proposed algorithm is calculated as

$$
\mathbf{w}_{\mathrm{re}}=\mu_{\mathrm{re}} \hat{\mathbf{R}}_{\mathrm{re}}^{-1} \mathbf{a}\left(\hat{\theta}_{0}\right)
$$

Then, the output of the adaptive beamformer is calculated as 


$$
\mathbf{Z}=\mathbf{w}_{\mathrm{re}}^{H} \mathbf{Y}=\mathbf{w}_{\mathrm{re}}^{H} \hat{\mathbf{B}} \mathbf{X}
$$

Processing block diagram of the proposed algorithm is described in Fig. 1. The known parameters of this algorithm contain array geometry and a presumed SOI direction sector. Based on these parameters, the proposed method can suppress coherent mainlobe and sidelobe interferences when SOI appears in the training data. Moreover, the proposed method improves robustness of the beamformer from the two aspects. First, since the INCM reconstruction procedure converts a MPDR beamformer to a MVDR beamformer, the proposed method is less sensitive to SV error when SOI appears in the training data. Second, due to the high accuracy of IAA spatial spectrum, the presumed SOI direction error can be corrected.

\section{Simulation results}

In simulations, we consider a uniform linear array with $M=16$ omnidirectional antenna elements spaced half a wavelength. The SOI direction is $0^{\circ}$, and the mainlobe interference direction is $5^{\circ}$ with interference-to-noise ratio (INR) $5 \mathrm{~dB}$. Two sidelobe interferences impinging

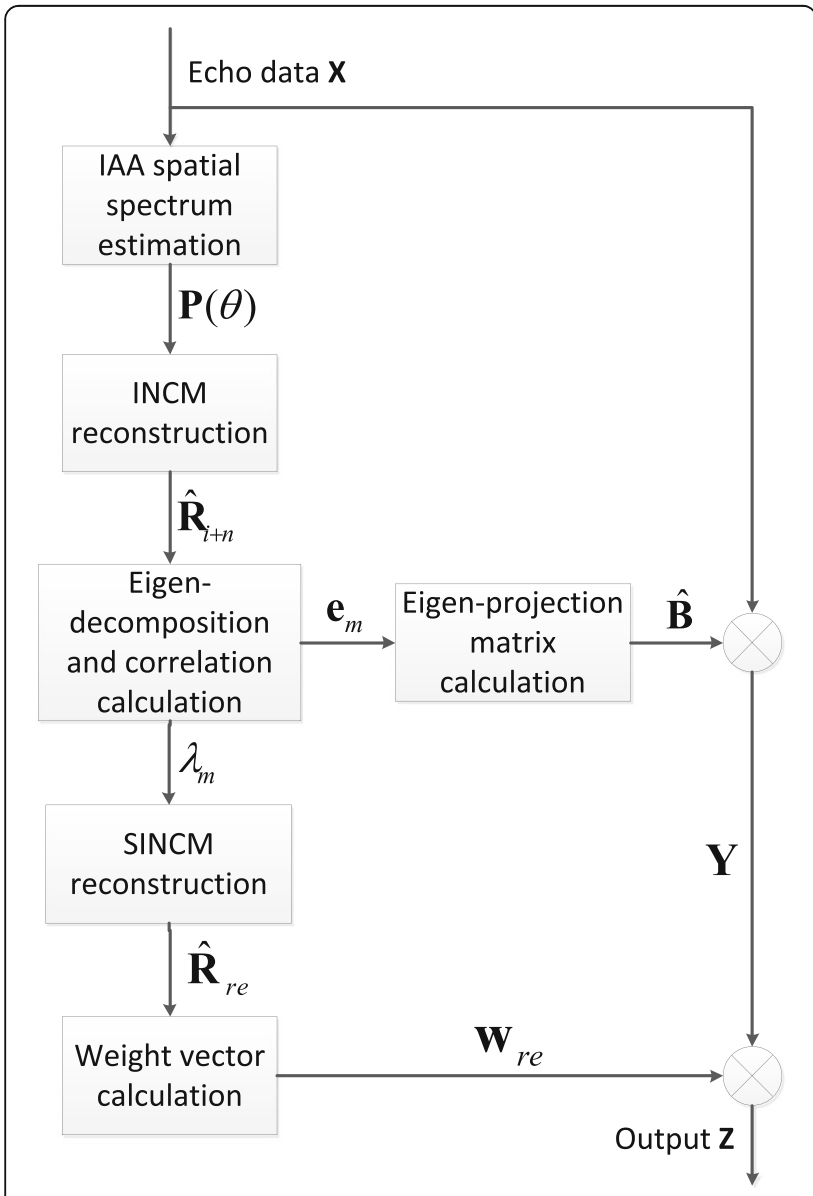

Fig. 1 Processing block diagram of the proposed algorithm from $-45^{\circ}$ to $35^{\circ}$ are considered with INR 25 and $35 \mathrm{~dB}$, respectively. Noise is assumed to be the Gaussian white additive noise. The number of snapshots is fixed to be $K=100$. The number of sidelobe interferences $\mathrm{Q}$ is assumed to be known in simulations. For each scenario, 200 Monte Carlo runs are performed.

The proposed method is compared to SMI, BMP, EMP, and the method proposed in [5] (EMP-CMR). The SOI is not present in the training data when using EMP and EMP-CMR methods. In BMP method, the exact mainlobe interference angle is known. For Capon and IAA, the scanning grid is uniform in the range from $-90^{\circ}$ to $90^{\circ}$ with $1^{\circ}$ increment between adjacent grid points. The presumed SOI angular sector is set as $\Theta=\left[-2^{\circ}, 2^{\circ}\right]$ in the proposed method.

First, the input signal signal-to-noise ratio (SNR) is fixed to be $0 \mathrm{~dB}$. We consider the accuracy of IAA, MUSIC, Capon, and forward-backward spatial smoothing (MSS) Capon spatial spectrum [16] when the SOI and the interference are coherent. When using the forward-backward spatial smoothing method, the array is divided into 2 sub-arrays and each sub-array contains 15 sensors. In Fig. 2a, the SOI and all the interferences are uncorrelated. In Fig. $2 \mathrm{~b}$, only the SOI and the mainlobe interference are coherent. In Fig. 2c, the SOI, the mainlobe interference, and the sidelobe interference from $-45^{\circ}$ are coherent. Figure 2 shows that IAA spatial spectrum outperforms the MUSIC and Capon spatial spectrum when coherent interference exists. Besides, the noise floor of the MSS Capon spatial spectrum is significantly higher than the IAA spatial spectrum.

Next, we compare the output signal-to-interferenceplus-noise (SINR) of different methods when input signal SNR varies. The presumed SOI direction is $0^{\circ}$. Figure 3 shows that EMP-CMR method outperforms other methods when all the sources are uncorrelated. Besides, the output SINR of the proposed method is better than BMP, EMP, and SMI methods. Figure 4 presents the output SINR when the SOI and the mainlobe interference are coherent. For EMP and EMP-CMR methods, the SOI is absent in the training data. Thus, the adaptive weighted vectors of these two methods are not affected by the coherent signals in this scenario. From Figs. 3 and 4, we observe that the performance of EMP and EMP-CMR remains unchanged. However, for the proposed method, its output SINR improves a lot due to better accuracy of IAA spatial spectrum. In Fig. 5, the adaptive array patterns of the quiescent (QUI), EMP-CMR, and the proposed method are depicted when SNR is $0 \mathrm{~dB}$. We note that the mainlobe peak offset is eliminated in both EMP-CMR method and the proposed method. Figure 6 shows the output SINR when the SOI, the mainlobe interference, and the sidelobe interference impinging from $35^{\circ}$ are all coherent. For EMP and EMP-CMR methods, the 

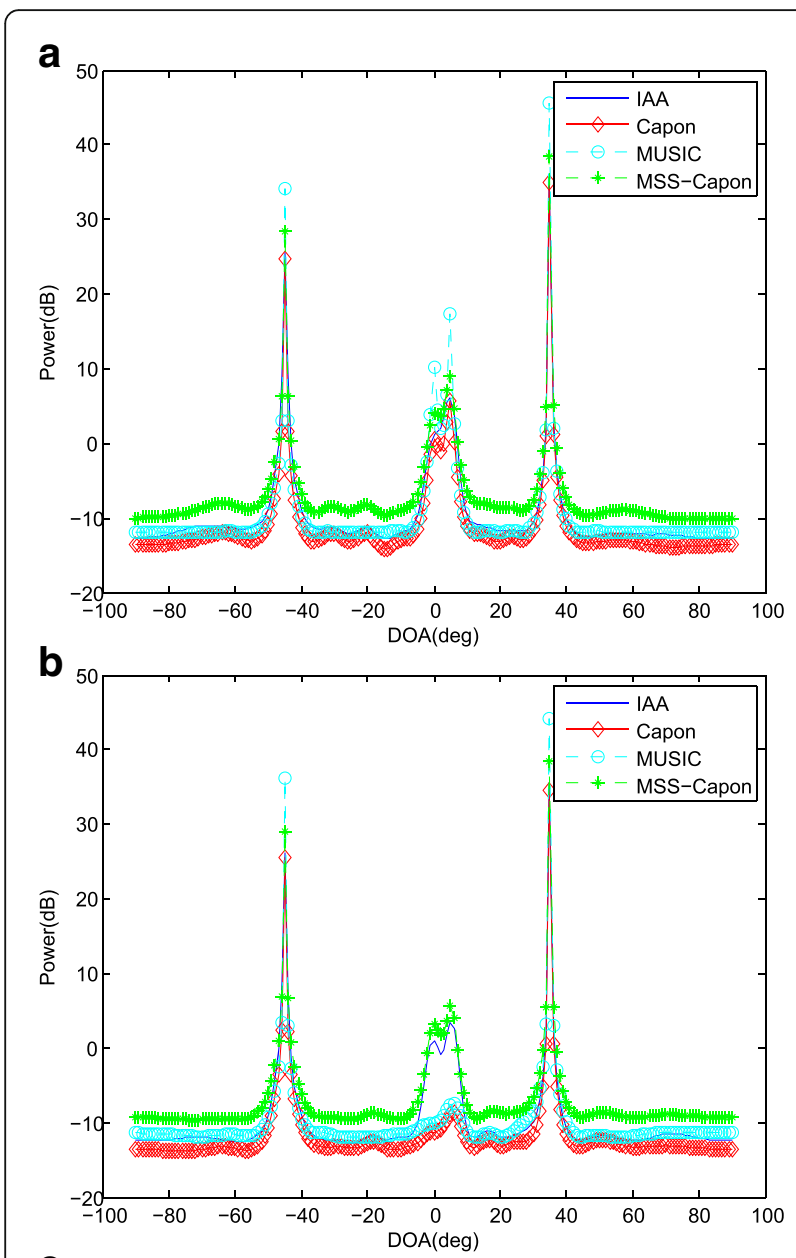

C

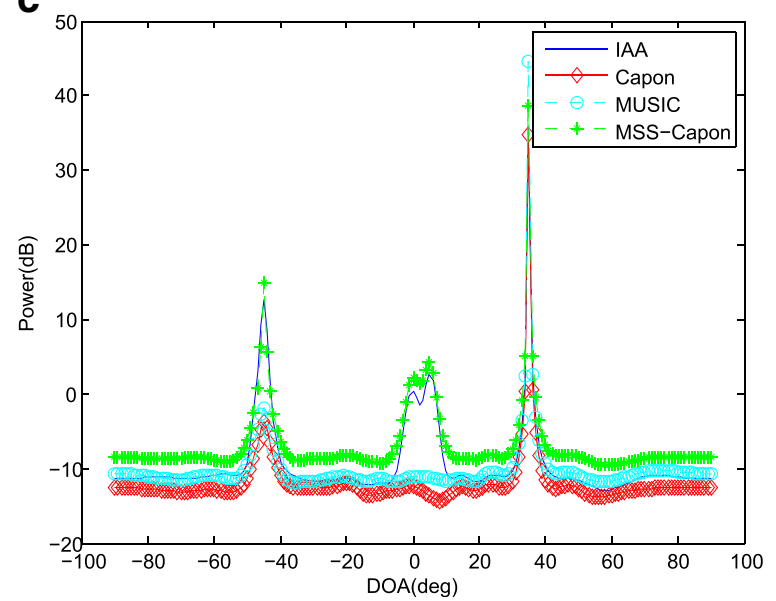

Fig. 2 Spatial spectrum under different circumstances. a SOI and interferences are uncorrelated. $\mathbf{b} \mathrm{SOI}$ and the mainlobe interference are coherent. c SOI, the mainlobe interference, and one sidelobe interference are coherent

calculation of adaptive weight vector is affected by the coherent sidelobe interferences. Therefore, the performance of these two methods degrades severely in Fig. 6. For the proposed method, its performance also degrades.

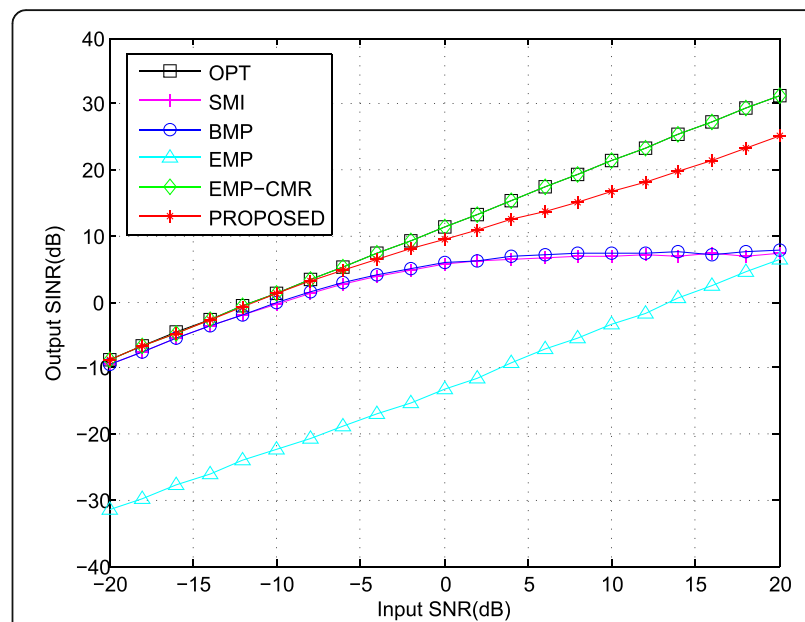

Fig. 3 Output SINR against input SNR for the case of uncorrelated sources

However, the output SINR of the proposed method is obviously higher than other methods in this scenario. Therefore, the proposed method has the best stability in complex coherent interference environment.

Finally, we examine the robustness of the beamformers to array SV error. We consider the SVs of the SOI and interferences to be randomly distributed in an uncertainty set [17], so it can be modeled as

$$
\mathbf{a}_{q}=\hat{\mathbf{a}}_{q}+\mathbf{e}_{q}
$$

where $\hat{\mathbf{a}}_{q}$ is the nominal SV of the direction $\hat{\theta}_{q}$ and $\mathbf{e}_{q}$ represents random SV error. $\mathbf{e}_{q}$ is set as follows:

$$
\mathbf{e}_{q}=\frac{\varepsilon_{q}}{\sqrt{M}}\left[e^{j \phi_{0}^{q}} e^{j \phi_{1}^{q} \cdots e^{j \phi_{M-1}^{q}}}\right]
$$

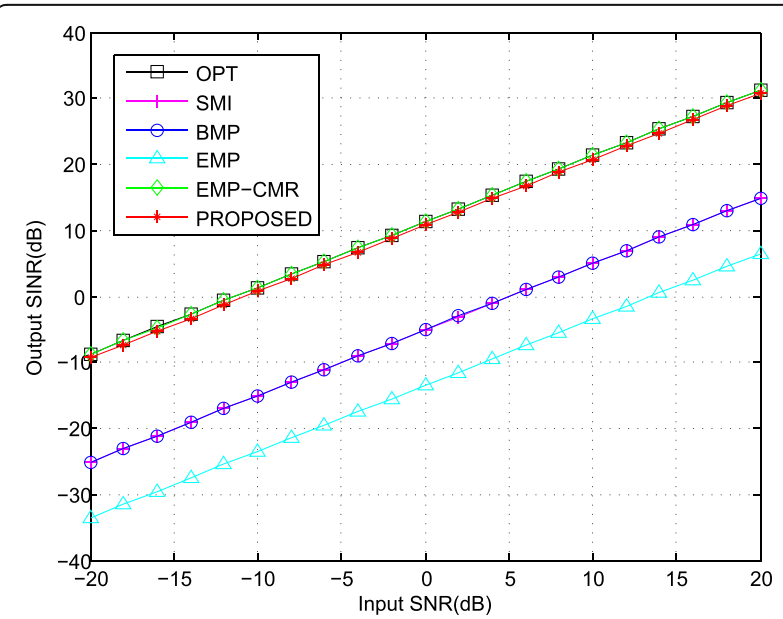

Fig. 4 Output SINR against input SNR for the case of two coherent sources 

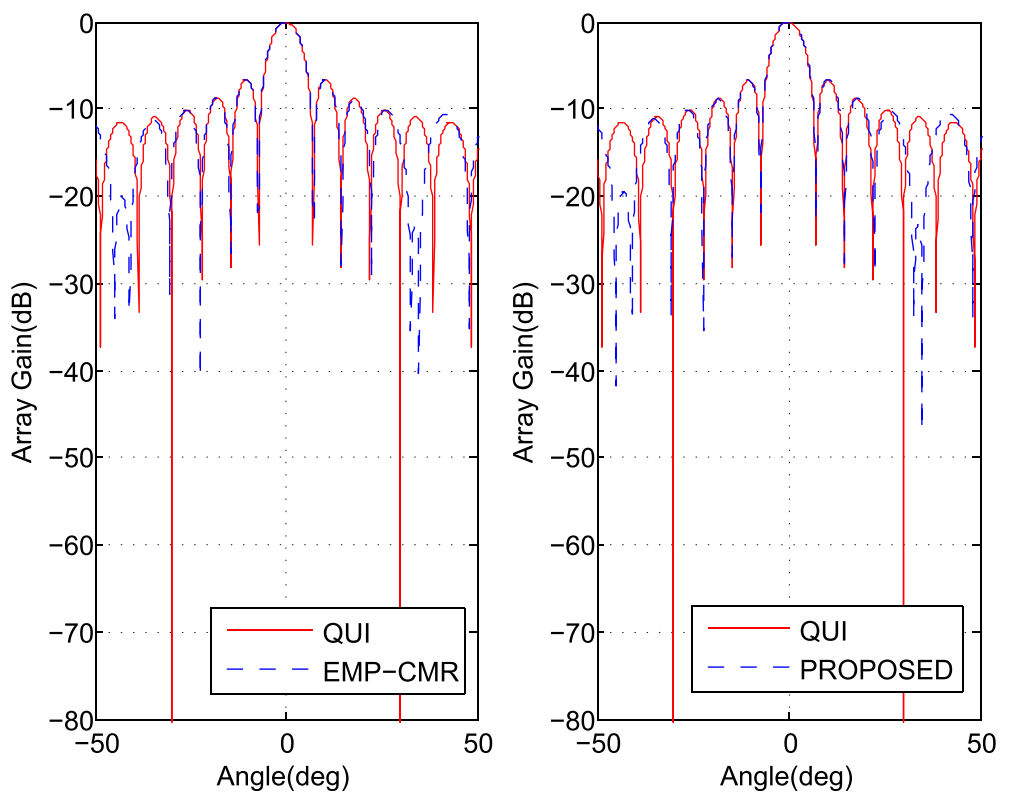

Fig. 5 Adaptive array patterns of QUI, EMP-CMR, and proposed method

where $\varepsilon_{q}$ denotes the norm of $\mathbf{e}_{q}$ and is uniformly distributed in the interval $[0,0.4]$ in each simulation run and $\phi_{m}^{q}(m=1,2, \cdots, M)$ is phases of $m$ th coordinate of the random error vector $\mathbf{e}_{q}$, which are independently and uniformly taken from the interval $[0,2 \pi]$ in each simulation run. $\hat{\theta}_{q}$ is uniformly distributed in the interval $\left[-2^{\circ}, 2^{\circ}\right]$ in each simulation run. This kind of SV mismatch model is universal because it can be caused by calibration error, local scattering error, look direction error, and so on. The other simulation conditions are the same to the simulation sets of Fig. 4. The output SINR versus the input SNR is shown in Fig. 7. From Fig. 7, we notice that the SV error has more side effects

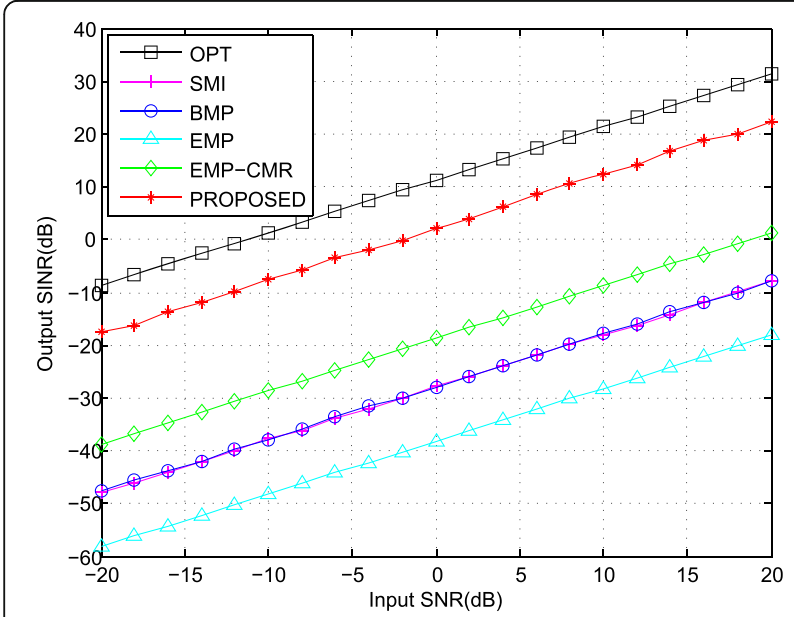

Fig. 6 Output SINR against input SNR for the case of three coherent sources on EMP-CMR method and the proposed method. However, the performance of EMP-CMR method and the proposed method is still better than other methods.

\section{Conclusions}

In this paper, we have developed a new mainlobe interference suppression method based on IAA spatial spectrum, INCM reconstruction, eigen-projection processing, and SINCM reconstruction, which annihilates the prior information restrictions existing in the previous methods for mainlobe interference suppression. Vigorous steps have been taken to reduce these restrictions, and simulation results demonstrated that the proposed method exhibits superior performance when coherent

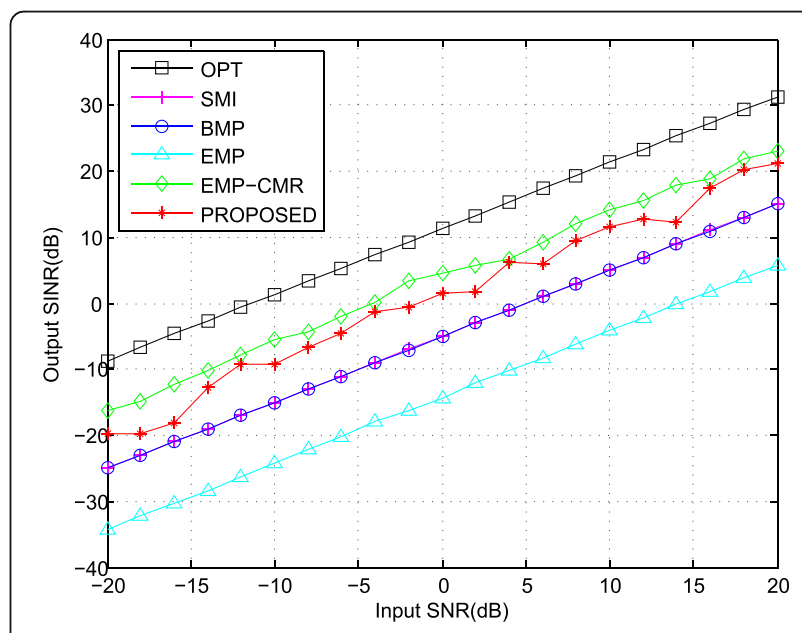

Fig. 7 Output SINR against input SNR for the case of SV random error 
mainlobe interference and sidelobe interferences exist. In addition, robustness of the proposed method against $\mathrm{SV}$ error is also verified in this paper.

\section{Acknowledgements}

This work was supported in part by the National Natural Science Foundation of China under Grant 61401489

\section{Competing interests}

The authors declare that they have no competing interests.

Received: 5 March 2016 Accepted: 1 December 2016

Published online: 13 December 2016

\section{References}

1. W Liu, J Liu, L Wang, K Duan, Z Chen, Y Wang, Adaptive array detection in noise and completely unknown jamming. Digital Signal Processing. 46 41-48 (2015)

2. W Liu, J Liu, X Hu, Z Tang, L Huang, Y Wang, Statistical performance analysis of the adaptive orthogonal rejection detector. IEEE Signal Processing Letters. 23(6), 873-877 (2016)

3. RF Li, YL Wang, SH Wan, Robust adaptive beam forming under main lobe interference conditions. System Engineering and Electronics. 24(7), 4-7 (2002)

4. RF Li, YL Wang, SH Wan, Research of reshaping adapted pattern under mainlobe interference conditions. Modern Radar. 24, 50-55 (2002)

5. XP Yang, Z Zhang, T Zeng, T Long, TK Sarkar, Mainlobe interference suppression based on eigen-projection processing and covariance matrix reconstruction. IEEE Antennas Wireless Propag. Lett. 13, 1369-1372 (2014)

6. J Qian, Z He, Mainlobe interference suppression with eigenprojection algorithm and similarity constraints. Electronics Lett. 52, 228-230 (2016)

7. SA Vorobyov, Principles of minimum variance robust adaptive beamforming design. Signal Process. 93, 3264-3277 (2013)

8. Special issue on passive radar systems-IEE Proceedings on Radar, Sonar and Navigation. 152(3), 106-223 (2005)

9. Y Zhao, R Li, Y Wang, W Liu, Angles measurement of meter-wave radars by mainlobe multipath jamming suppression. J. Huazhong Univ. of Science and Technology (Natural Science Edition) 41(4), 51-55 (2013)

10. Y Gu, A Leshem, Robust adaptive beamforming based on interference covariance matrix reconstruction and steering vector estimation. IEEE Signal Process. Mag. 60(7), 2974-2987 (2012)

11. J Liu, H Li, B Himed, Joint optimization of transmit and receive beamforming in active arrays. IEEE Signal Process Lett. 21(1), 39-42 (2014)

12. J Liu, W Liu, H Liu, B Chen, XG Xia, F Dai, Average SINR calculation of a persymmetric sample matrix inversion beamformer. IEEE Signal Process. Mag. 64(8), 2135-2145 (2016)

13. T. Yardibi, J. Li, P. Stoica, M. Xue, A.B. Baggeroer. Source localization and sensing: a nonparametric iterative adaptive approach based on weighted least squares. IEEE Trans. Aerospace and Electronic Systems. (2008)

14. L Du, T Yardibi, J Li, P Stoica, Review of user parameter-free robust adaptive beamforming algorithms. Digital Signal Process. 19(4), 567-582 (2009)

15. L. Ehrenberg, S. Gannot A. Leshem, E. Zehavai. Sensitivity analysis of MVDR and MPDR beamformers. IEEE 26th Convention of Electr. Electron. Eng. in Israel, Eliant, Israel, Nov., 2010.

16. W Liu, L Zhang, Robust beamforming for coherent signals based on the spatial-smoothing technique. Signal Process. 92(11), 2747-2758 (2012)

17. L Huang, XX Zhang, Z Ye, Robust adaptive beamforming with a nove interference-plus-noise covariance matrix reconstruction method. IEEE Trans. Signal Process. 63, 1643-1650 (2015)

\section{Submit your manuscript to a SpringerOpen ${ }^{\circ}$ journal and benefit from:}

- Convenient online submission

- Rigorous peer review

- Immediate publication on acceptance

- Open access: articles freely available online

- High visibility within the field

- Retaining the copyright to your article

Submit your next manuscript at $>$ springeropen.com 